\title{
Strain rate dependent shear localization and deformation mechanisms in the CrMnFeCoNi high-entropy alloy with various microstructures
}

\author{
Zhengling Yang a,b,1, Muxin Yang ${ }^{\mathrm{a}, 1}$, Yan Ma ${ }^{\mathrm{a}, \mathrm{b}}$, Lingling Zhou ${ }^{\mathrm{a}}$, Wenqiang Cheng ${ }^{\mathrm{a}, \mathrm{b}}$, \\ Fuping Yuan ${ }^{\mathrm{a}, \mathrm{b}, *}$, Xiaolei $\mathrm{Wu}^{\mathrm{a}, \mathrm{b}}$ \\ ${ }^{a}$ State Key Laboratory of Nonlinear Mechanics, Institute of Mechanics, Chinese Academy of Science, 15 Beisihuan West Road, Beijing, 100190, China \\ ${ }^{\mathrm{b}}$ School of Engineering Science, University of Chinese Academy of Sciences, 19A Yuquan road, Beijing, 100049, China
}

\section{A R T I C L E I N F O}

\section{Keywords:}

High entropy alloys

Deformation twins

Shear localization

Strain rate effect

Strain hardening

\begin{abstract}
A B S T R A C T
CrMnFeCoNi high-entropy alloys (HEA) with various microstructures have been produced using cold rolling followed by critical annealing at various temperatures. Shear deformation behaviors of this HEA with various microstructures at a wide range of strain rates $\left(2 \times 10^{-3}-5 \times 10^{4} \mathrm{~s}^{-1}\right)$ have been characterized using hat-shaped specimens. Strain hardening exponent and strain rate sensitivity have been obtained for various microstructures. No shear localization was observed up to shear strain of 8 for all microstructures under lower strain rates $\left(2 \times 10^{-}\right.$ $\left.{ }^{3}-1 \times 10^{1} \mathrm{~s}^{-1}\right)$, while stress drop and shear localization were found to occur at various critical shear strains for various microstructures under dynamic shear loading $\left(5 \times 10^{4} \mathrm{~s}^{-1}\right)$. A new formula, considering competition of strain hardening and strain rate hardening against thermal softening, was proposed to estimate the critical shear strains under dynamic shear loading and the predicted results were found to be in a fairly good agreement with the experimental data. Based on micro-hardness testing, the strain hardening due to the microstructure evolution was found be much stronger under dynamic shear loading than that under quasi-static loading at the same interrupted shear strain, which can be attributed to the more efficient grain refinement and the triggered hierarchical deformation nanotwins under dynamic shear deformation.
\end{abstract}

\section{Introduction}

Equal-molar multi-component high-entropy alloys (HEAs) [1-19] and medium-entropy alloys (MEAs) [20-34] have attracted extensive research interests recently due to their excellent mechanical properties, especially under cryogenic temperature. This class of materials are typically solid solutions with a single crystal structure, with exceptions with dual-phase structure [12] or precipitates [14-16,33]. The random distribution of multiple elements for HEAs or MEAs with FCC structure can result in low stacking fault energy (SFE) in these alloys [2-11, 20-31]. As a result, deformation twinning is the dominant deformation mechanism (especially under cryogenic temperature) in this type of alloys, such as CrMnFeCoNi HEA [2-11] and CrCoNi MEA [20-31].

As we know, HEAs and MEAs with coarse grains (CG) have a relatively low yield strength, which limits their structural applications. Cold working and grain refinement by severe plastic deformation (SPD) have been proven to be effective methods to improve the yield strength in metals and alloys, while the tensile ductility is usually sacrificed at the same time [22-24]. In last two decades, heterogeneous structures, in which different domains with dramatic difference in mechanical properties exist, have been proven to be an effective strategy to achieve simultaneous high strength and appreciable ductility [24,35-37]. This strategy has also been applied to HEAs and MEAs recently for obtaining excellent synergy of strength and ductility [24,25,27]. Moreover, the tensile behaviors and the strain hardening mechanisms of HEAs and MEAs with heterogeneous structures under quasi-static conditions have been well documented [2-11,20-24,27].

It is well known that the mechanical behaviors of metals and alloys under high-strain-rate impact loading are dramatically distinct from those under quasi-static conditions due to the strain rate and adiabatic thermal softening effects [38-41]. Recently, dynamic behaviors and strain rate effects on mechanical properties for HEAs and MEAs with

\footnotetext{
* Corresponding author. State Key Laboratory of Nonlinear Mechanics, Institute of Mechanics, Chinese Academy of Science, 15 Beisihuan West Road, Beijing, 100190, China.

E-mail addresses: yangzhengling@imech.ac.cn (Z. Yang), mxyang@lnm.imech.ac.cn (M. Yang), mayan@imech.ac.cn (Y. Ma), 1lzhou@lnm.imech.ac.cn (L. Zhou), vestentceng@qq.com (W. Cheng), fpyuan@lnm.imech.ac.cn (F. Yuan),xlwu@imech.ac.cn (X. Wu).

1 These two authors contributed equally to this work.
} 
homogeneous structures have been extensively investigated since the fundamental understanding of dynamic deformation mechanisms are crucially important for the applications of HEAs and MEAs in the crash relevant and impact-tolerant structures [42-56]. For example, the deformation behaviors under dynamic loading for a single phase FCC CrMnFeCoNi HEA with CG have been studied using hat-shaped specimens in Hopkinson bar [42]. This CrMnFeCoNi HEA with CG has been found to show high dynamic ductility due to the excellent strain hardening ability and moderate thermal-softening effect. The compressive deformation behaviors for the CrMnFeCoNi HEA with CG in a wide range of strain rates $\left(10^{-4}-10^{4} \mathrm{~s}^{-1}\right)$ have been investigated [43], and the strain rate sensitivity (SRS) and the strain hardening mechanisms have been studied, and finally the modified Johnson-Cook model with fitted parameters by experiments results has been proposed. Unlike the dominant deformation mechanism by dislocation slip under quasi-static conditions in the HEAs/MEAs with CG, deformation twinning has been observed to be triggered and contribute an important part for the total plastic strain under dynamic loading due to the elevated flow stress [25, $28,31,43]$.

The dynamic behaviors of HEAs and MEAs with heterogeneous structures might be totally different from those for HEAs and MEAs with CG since both CG and ultrafine grains (UFG) exist simultaneously in the heterogeneous grain structure [24,25,27]. Although the dynamic shear mechanisms for a single phase FCC CrCoNi MEA with heterogeneous structures have been uncovered in our recent work [25], the strain rate and thermal softening effects, and the possible deformation mechanisms at various strain rates are still not well documented for HEAs and MEAs with heterogeneous structures. In this regard, using a single phase FCC CrMnFeCoNi HEA as model material, the shear deformation behaviors of HEAs with various microstructures in a wide range of strain rates $(2 \times$ $10^{-3}-5 \times 10^{4} \mathrm{~s}^{-1}$ ) have been systematically studied using hat-shaped specimens and subsequent microstructure observations in the present study.

\section{Materials and experimental procedures}

The equal-molar CrMnFeCoNi HEA was melted under a high-purity argon atmosphere by electromagnetic levitation, and then cast into ingots. The resultant ingots have an actual chemical composition of 19.91Cr-19.36Mn-20.15Fe-20.07Co-19.23Ni-1.28others (in wt. \%). The other impurities (1.28 in wt. \%) include sulfur $(<700 \mathrm{ppm})$ and phosphorus $(<100 \mathrm{ppm})$, as well as other possible impurity elements introduced by the raw materials. Moreover, the chemical composition in atomic ratio is $21.70 \mathrm{Cr}-19.97 \mathrm{Mn}-20.45 \mathrm{Fe}-19.30 \mathrm{Co}-18.58 \mathrm{Ni}$, without considering the impurities. The ingots were first annealed for homogenization at $1473 \mathrm{~K}$ for $24 \mathrm{~h}$, and then hot-forged into plates with a thickness of $5 \mathrm{~mm}$ between $1423 \mathrm{~K}$ and $1173 \mathrm{~K}$. Next, the plates were cold-rolled (CR) by a thickness reduction of $80 \%$ (the CR plates have a final thickness of about $1 \mathrm{~mm}$ ). In order to obtain varying heterogeneous microstructures, the CR plates were annealed at various temperatures $(848,873,948,973,1073 \mathrm{~K})$ for $1 \mathrm{~h}$, immediately followed by water quenching.

Before shear testing, the quasi-static uniaxial tensile behaviors for varying heterogeneous microstructures have been characterized under displacement control by using an Instron 5565 testing machine at a strain rate of $5 \times 10^{-4} \mathrm{~s}^{-1}$. The tensile direction was set to be parallel to the rolling direction, an extensometer was utilized to measure the displacement during the tensile testing. Shear deformation testing has been conducted on hat-shaped specimens in a wide range of strain rates $\left(2 \times 10^{-3}-5 \times 10^{4} \mathrm{~s}^{-1}\right)$. The testing in the range of $\left(2 \times 10^{-3}-1 \times 10^{1} \mathrm{~s}^{-1}\right)$ was performed using MTS Landmark testing machine, and the high strain rate testing $\left(5 \times 10^{4} \mathrm{~s}^{-1}\right)$ was performed using a split-Hopkinson pressure bar (SHPB). The hat-shaped plate specimens were machined from the plates with the loading direction parallel to the rolling direction. The geometry and dimensions of the hat-shaped specimen and specimen holder, and the configuration for SHPB and dynamic shear testing are shown in Fig. 1. The specimen holders made of high strength maraging steel were used to ensure pure shear deformation in the plated hat-shaped specimens by preventing the lateral expansion of two legs and stop the dynamic shear testing at the pre-determined shear displacement for subsequent microstructure observations in the interrupted "frozen" experiments. The dynamic shear testing can be stopped at pre-determined shear displacements by controlling the height of the high strength maraging steel due to the much larger contact area of the holder. The other details about the dynamic shear testing using plated hat-shaped specimens in Hopkinson-bar can be referred to our previous paper [25]. The same specimen and specimen holder (Fig. 1a) are utilized in the lower strain rate shear testing by MTS Landmark testing machine. For both tensile and shear testing, three tests were conducted for each specimen to check the repeatability. Electron backscattered diffraction (EBSD) and transmission electron microscope (TEM) have been utilized to reveal the microstructures before and after shear testing at various strain rates. The sample surfaces for EBSD were first mechanically polished, and then polished with a $0.05 \mu \mathrm{m} \mathrm{SiO} 2$ aqueous suspension, followed by electro-polishing in a solution of 5\% $\mathrm{HClO} 4$ and $95 \%$ alcohol at $37 \mathrm{~V}$ and $253 \mathrm{~K}$. For TEM observations, thin disks were first mechanically polished to about $40 \mu \mathrm{m}$, followed by a twin-jet polishing using a solution of 5\% perchloric acid and 95\% ethanol at $25 \mathrm{~V}$ and $253 \mathrm{~K}$. Micro-hardness distributions in the shear zone and the hardness increments before and after shear testing for various samples were characterized on the polished samples using a Vickers diamond indenter at a load of $5 \mathrm{~g}$ for $15 \mathrm{~s}$ dwell time. Three group of tests were conducted for each point to obtain the average micro-hardness for each position.

\section{Results and discussions}

The microstructures prior to mechanical testing for the CR sample and the samples annealed at various temperatures (848, 873, 948, 973, $1073 \mathrm{~K}$ ) are shown in Figs. 2 and 3. Fig. 2 shows the EBSD (inverse pole figure, IPF) images for various samples. The samples annealed at 848 , $873,948 \mathrm{~K}$ have heterogeneous microstructures consisting of both recrystallized CG and retained UFG. Therefore, in Fig. $2 \mathrm{~b}-\mathrm{d}$ for the samples with heterogeneous microstructures, only the distributions of recrystallized CG are displayed by blacking out UFG areas. The CR sample has a typical deformed lamellar structure (Fig. 2a). It is interesting to note that the area fraction of the recrystallized CG increases with increasing annealing temperature for the samples with heterogeneous microstructures. For example, the area fraction of the recrystallized CG is increased from $15.2 \%$ to $30.3 \%$ and to $48.5 \%$ in the samples annealed at $848,873,948 \mathrm{~K}$. The average grain size for the recrystallized CG also increases with increasing annealing temperature for the samples with heterogeneous microstructures. However, the samples annealed at 973 and $1073 \mathrm{~K}$ show fully recrystallized microstructures (Fig. 2e-f), and the average grain sizes are 6.87 and $7.90 \mu \mathrm{m}$, respectively. The average grain sizes were estimated by intercept method based on at least ten lines (more than 100 grains). In the samples annealed at 973 and $1073 \mathrm{~K}$, numerous annealing twins are also shown.

TEM images for one typical heterogeneous microstructure (annealed at $873 \mathrm{~K}$ ) and one typical fully recrystallized microstructure (annealed at $1073 \mathrm{~K}$ ) are displayed in Fig. 3a-b and 3c-3d, respectively. As indicated in the TEM bright-field image for the sample annealed at $873 \mathrm{~K}$, both the recrystallized CG area and the retained UFG area can be clearly observed. Sharp grain boundaries (GBs) are well identified for recrystallized CG and straight twin boundaries (TBs) for annealing twins can be clearly observed. Moreover, the density of dislocations is low in the interiors of the recrystallized CG, while the retained UFG area has high density of dislocations. The grain size for recrystallized CG area is about several microns, which is consistent with the previous EBSD results. However, only recrystallized large CG are identified for the sample annealed at $1073 \mathrm{~K}$, the dislocation density is very low for this sample and also a few annealing twins can be observed in the grain interiors. 

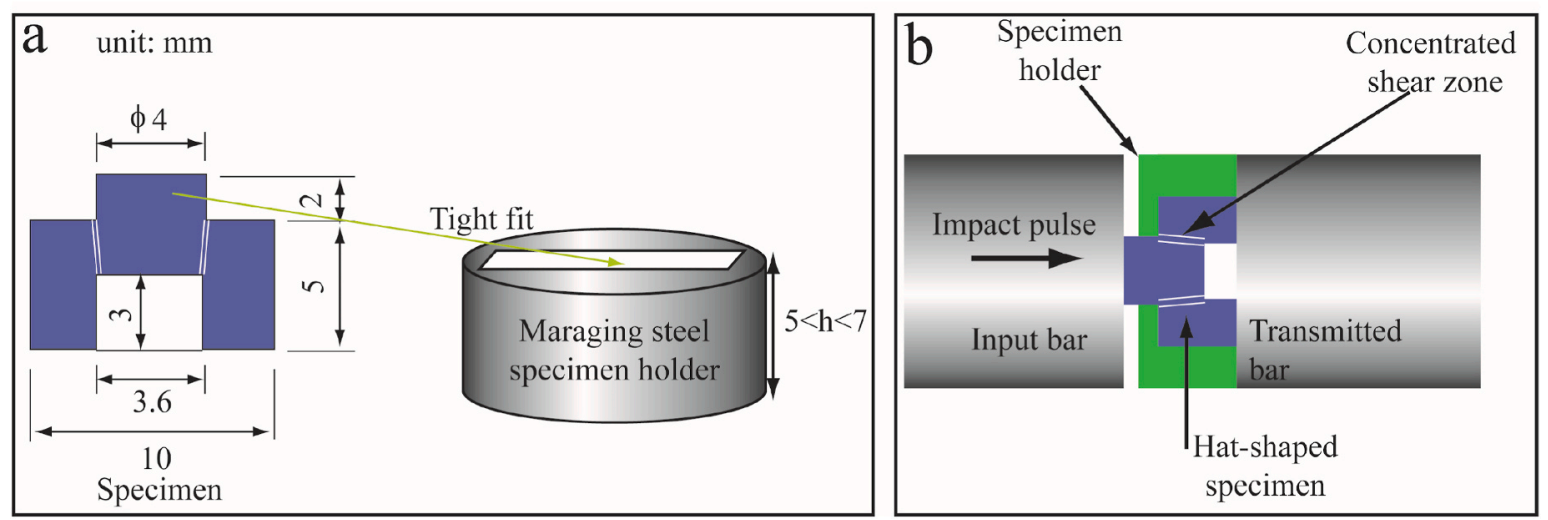

Fig. 1. (a) The geometry and dimensions of the hat-shaped specimen and specimen holder; (b) The configuration for SHPB and dynamic shear testing.

The average grain size for the sample annealed at $1073 \mathrm{~K}$ is obviously larger than that for the recrystallized CG in the sample annealed at 873 $\mathrm{K}$, which is consistent with the previous EBSD results.

The tensile properties for the CR sample and the samples annealed at various temperatures are shown in Fig. 4. The engineering stress-strain curves are shown in Fig. 4a, while the true stress-strain curves and the strain-hardening rate vs. true strain curves are displayed in Fig. 4b. The yield strength point and the ultimate strength (UTS) point (also called as the uniform elongation point, UE point) are marked in Fig. 4a. It is clearly indicated that the yield strength decreases while the UE increases with increasing annealing temperature. Moreover, the strain hardening rate is larger when annealed at higher temperatures. The well known Ludwik's equation can generally be used to describe the strain hardening behaviors of metals and alloys [57]:

$\sigma=\sigma_{0}+K \varepsilon^{n}$

where $\sigma_{0}$ is the yield strength, and $K$ is the strength coefficient. By fitting the experimental curves to this equation, the strain hardening exponent ( $n$ ) can be obtained for various samples, and the obtained $n$ index would be used later on for discussing the strain localization susceptibilities for the formation of adiabatic shear band (ASB) in various samples under high-strain-rate dynamic shear deformation.

The shear tests have been done under four different strain rates (about $2 \times 10^{-3}, 1 \times 10^{-1}, 1 \times 10^{1}$, and $5 \times 10^{4} \mathrm{~s}^{-1}$ ) for samples with various microstructures to show the strain rate effects on the
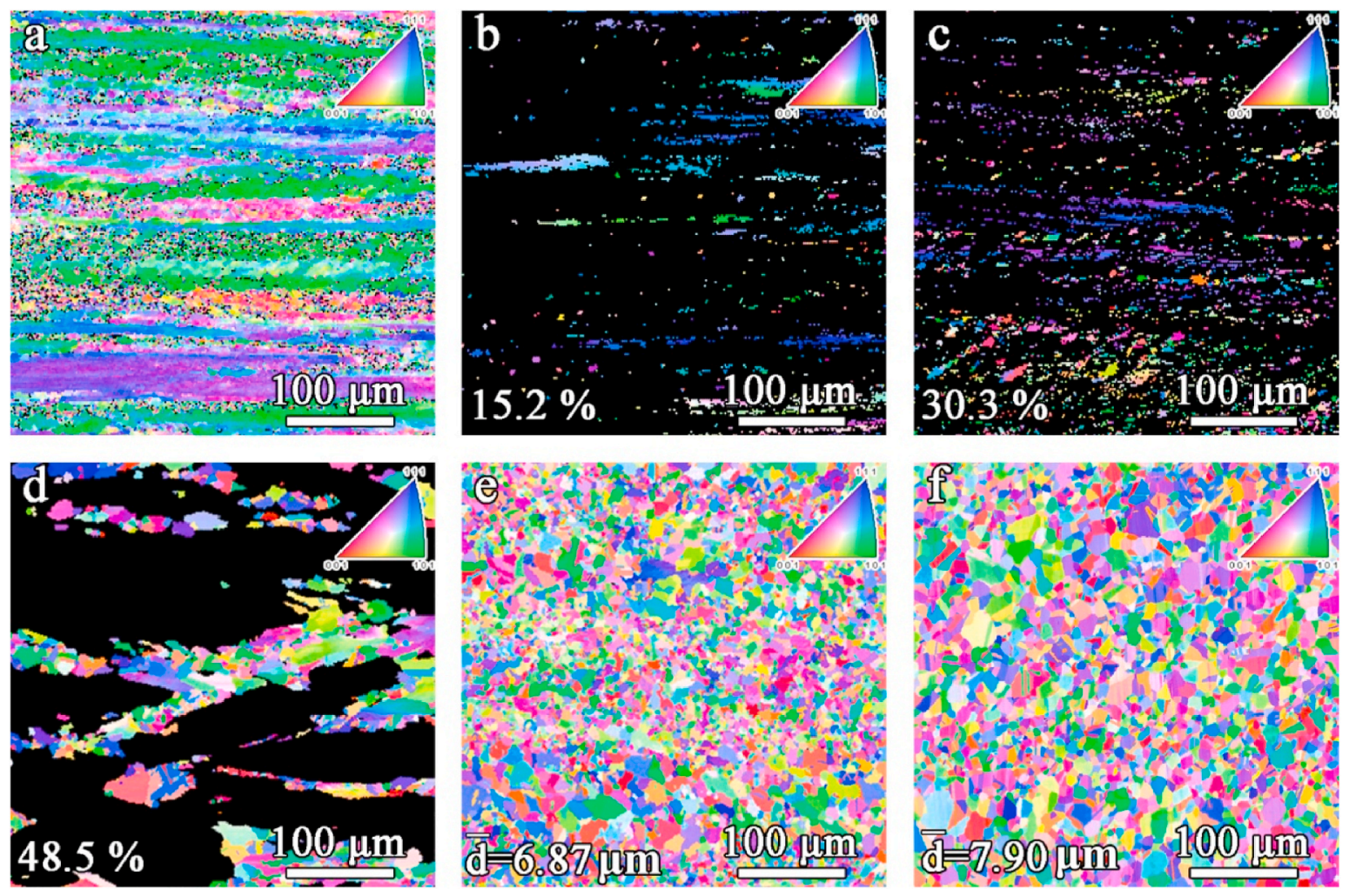

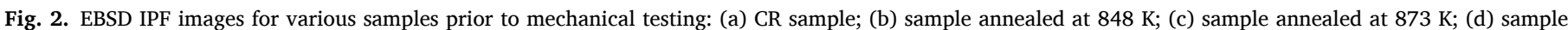

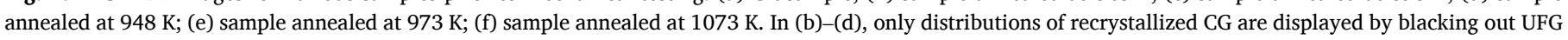
areas, and area fractions of recrystallized CG are also indicated. In (e)-(f), the average grain sizes are displayed. 

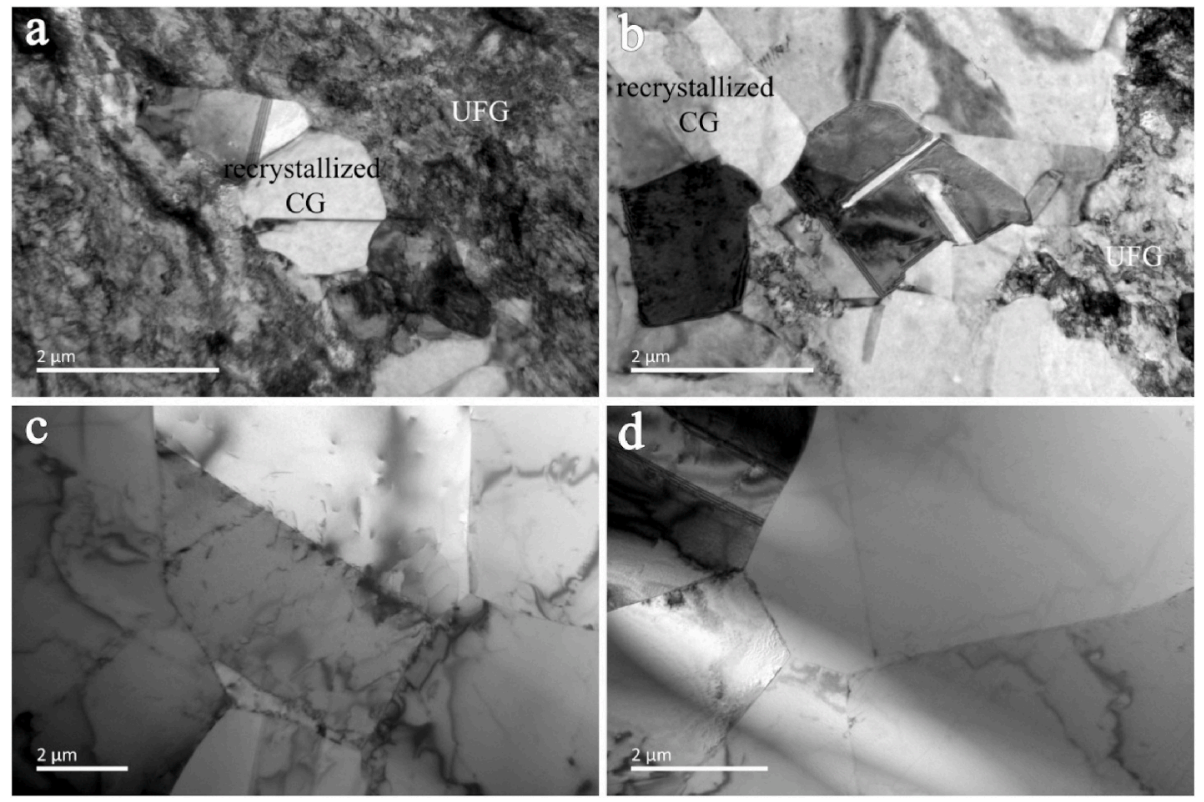

Fig. 3. TEM bright-field images for typical microstructures prior to mechanical testing: (a) and (b) one typical heterogeneous microstructure (annealed at $873 \mathrm{~K}$ ); (c) and (d) one typical fully recrystallized microstructure (annealed at $1073 \mathrm{~K}$ ). Figs. 5 and 6. In each figure of Fig. 5 (Fig. 5a-d), the shear stress-strain curves are plotted for various microstructures for each strain rate, respectively. As shown in Fig. 5, no strain localization is observed up to the shear strain of about 8 for various microstructures under the strain rates of $2 \times 10^{-3}, 1 \times 10^{-1}, 1 \times 10^{1} \mathrm{~s}^{-1}$. Under the strain rates of $2 \times 10^{-3}$ and $1 \times 10^{-1}$, the microstructures annealed at 873, 948, $1073 \mathrm{~K}$ show strong strain hardening, while the $\mathrm{CR}$ sample and the microstructure annealed at $848 \mathrm{~K}$ display slight strain hardening. Under the strain rate of $1 \times 10^{1} \mathrm{~s}^{-1}$, the microstructures annealed at $948,1073 \mathrm{~K}$ show strong strain hardening, while the CR sample and the microstructures annealed at 848 and $873 \mathrm{~K}$ display slight strain hardening. Obviously, the shear yield strength is observed to decrease, and the strain hardening ability is found to increase with increasing area fraction of recrystallized CG or with increasing annealing temperature. While, strain localization, such as shear band, occurs at various shear strains for various microstructures under the dynamic shear testing with the strain rate of about $5 \times 10^{4} \mathrm{~s}^{-1}$. The maximum stress points can generally be considered as the onset of the strain localization under dynamic shear loading [25], therefore the uniform shear strains for various microstructures under dynamic shear loading are found to increase with increasing annealing temperature.

While, in each figure of Fig. 6 (Fig. 6a-e), the shear stress-strain

curves are plotted for tests conducted under various strain rates for each microstructure, respectively. Thus, the strain rate effects on the flow behaviors of each microstructure can be analyzed. It is clearly shown that the shear yield strength increases with increasing strain rate for each microstructure. Moreover, the shear stress-strain curves under the dynamic shear testing with the strain rate of about $5 \times 10^{4} \mathrm{~s}^{-1}$ show much "apparent" lower strain hardening ability for each microstructure when compared to those conducted under the other lower strain rates, possibly due to the adiabatic heating and the thermal softening effect [25,40-42]. As shown later, the "actual" strain hardening due to the microstructure evolution (reflected by the hardness increment) is observed to be higher under high strain rate when compared to that under quasi-static strain rate. Based on these curves in Fig. 6, the SRS for each microstructure can be obtained.

Based on Eq. (1), the strain hardening exponent $(n)$ under the quasistatic uniaxial tensile testing can be obtained and is plotted as a function of annealing temperature in Fig. 7a. It is observed that the $n$ index increases almost linearly with the annealing temperature, indicating stronger strain hardening after higher temperature annealing. The SRS of flow stress for each microstructure can be obtained by the following equation:
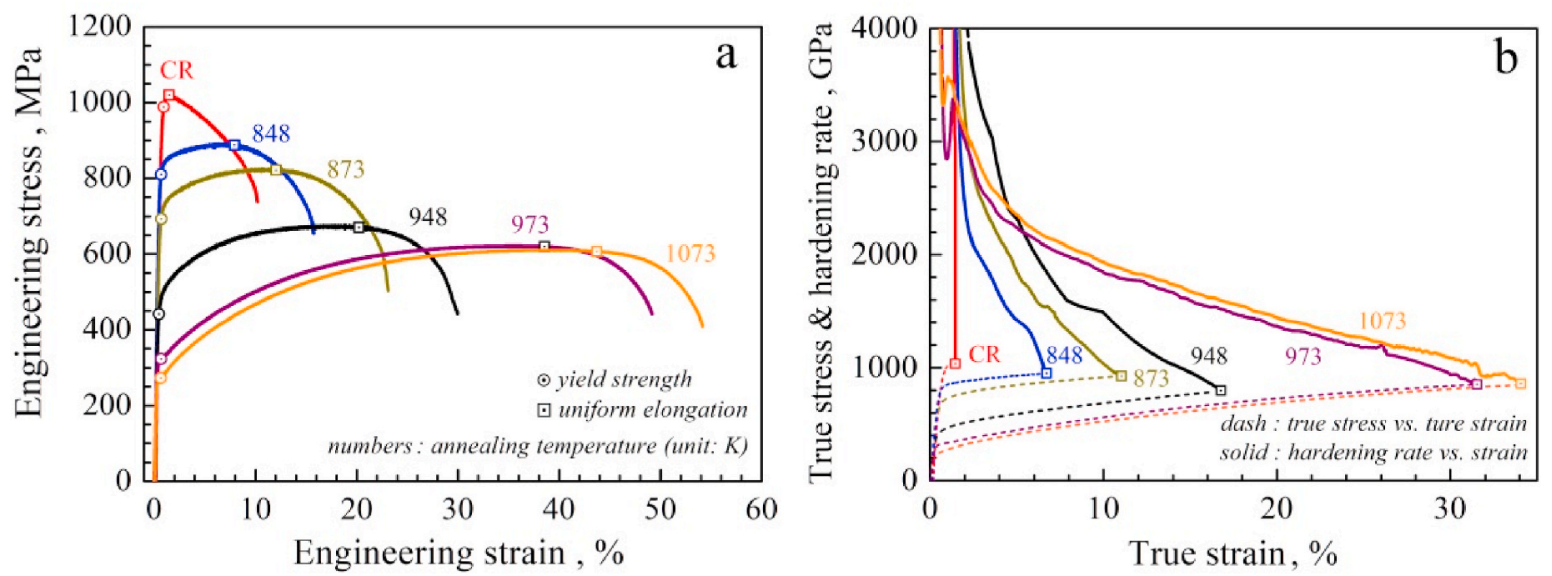

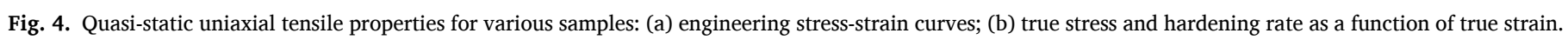



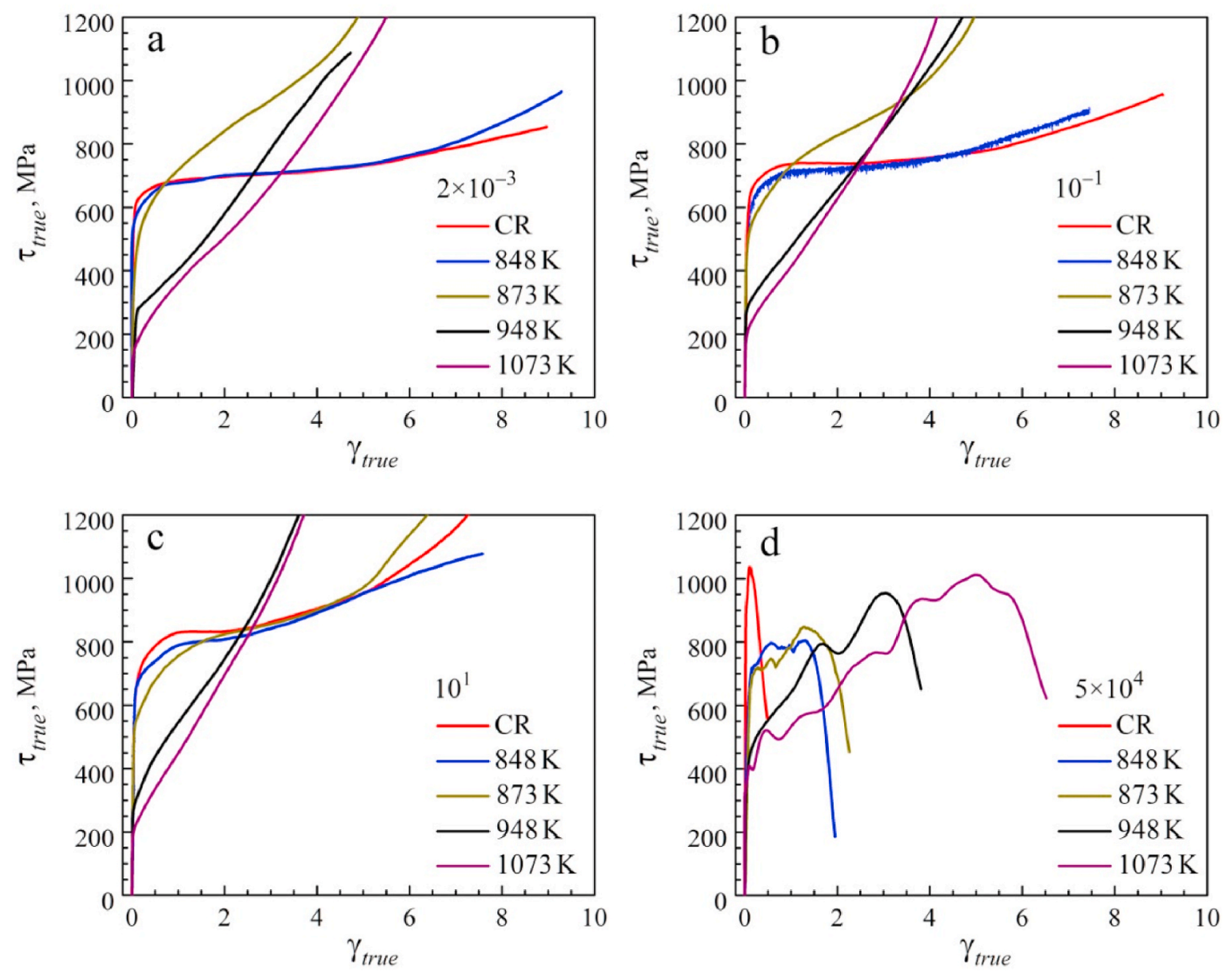

Fig. 5. Shear properties for various samples under four different strain rates: (a) $2 \times 10^{-3} \mathrm{~s}^{-1}$; (b) $1 \times 10^{-1} \mathrm{~s}^{-1}$; (c) $1 \times 10^{1} \mathrm{~s}^{-1}$; (d) $5 \times 10^{4} \mathrm{~s}^{-1}$.
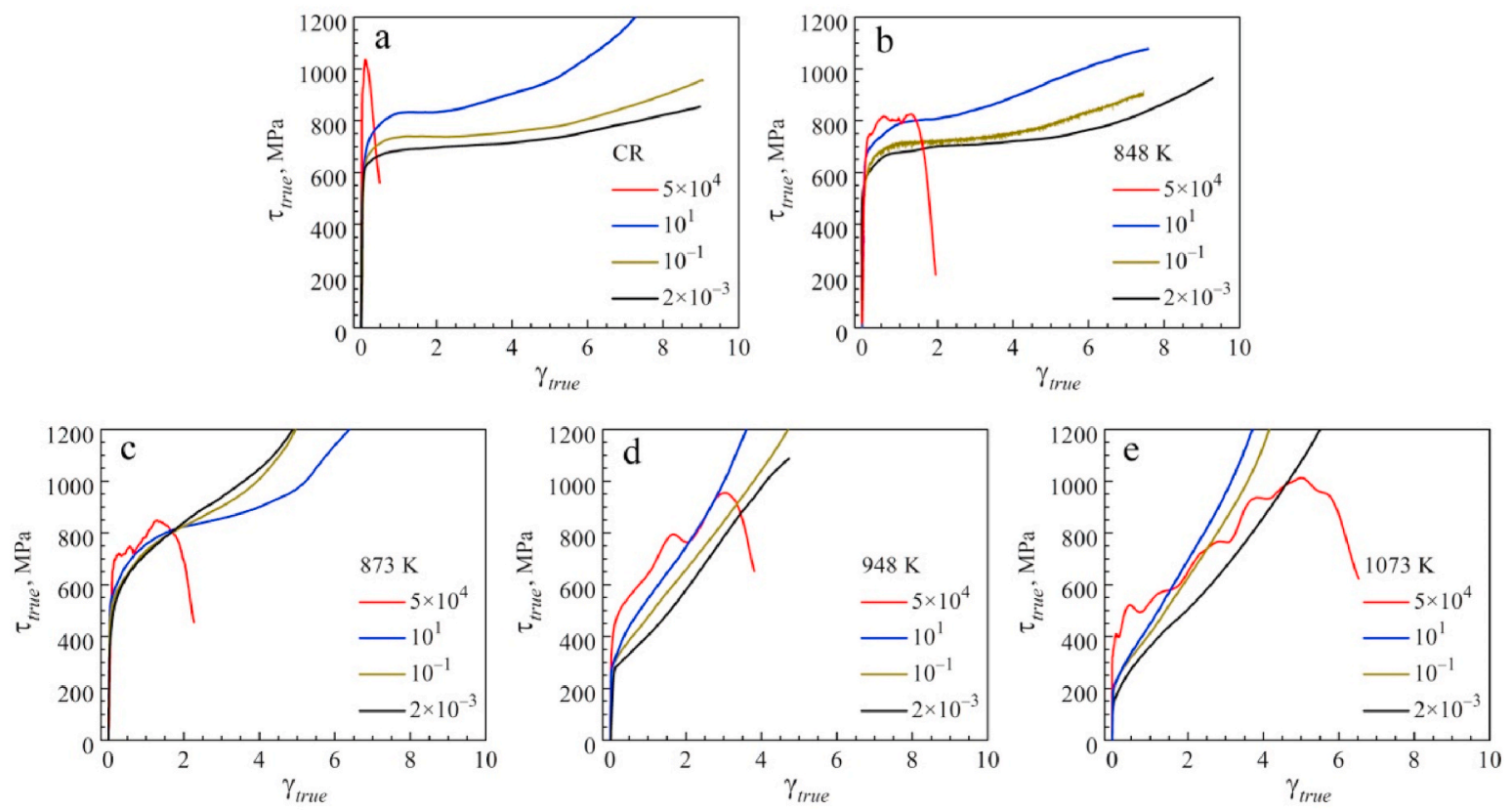

Fig. 6. Shear properties under different strain rates for five different microstructures: (a) CR sample; (b) sample annealed at $848 \mathrm{~K}$; (c) sample annealed at $873 \mathrm{~K}$; (d) sample annealed at $948 \mathrm{~K}$; (e) sample annealed at $1073 \mathrm{~K}$. 

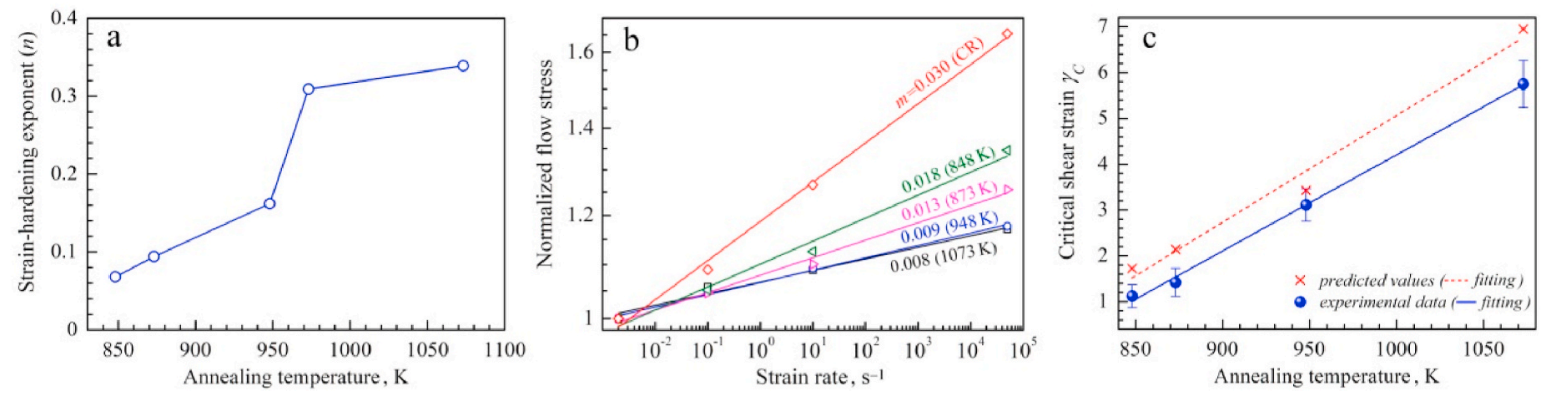

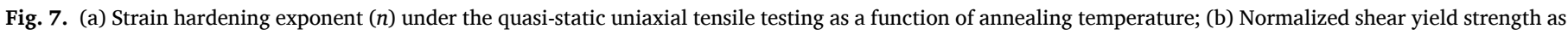

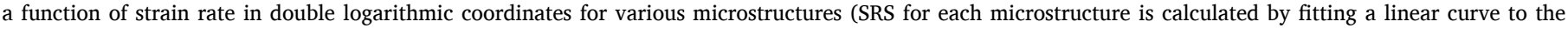
experimental data); (c) Experimental and predicted critical shear strains (Eq. (6)) for onset of shear localization as a function of annealing temperature.

$m=\left(\frac{\partial \ln \sigma}{\partial \ln \dot{\varepsilon}}\right)_{T, \varepsilon}$

Where $\sigma, \dot{\varepsilon}, \varepsilon, T$ are true stress, true strain rate, true strain and applied temperature, respectively. The normalized shear flow stress at the yielding point (divided by the yield stress at the strain rate of $2 \times 10^{-3} \mathrm{~s}^{-}$ ${ }^{1}$ ) is plotted as a function of strain rate in double logarithmic coordinates for various microstructures in Fig. 7b. Then the SRS for each microstructure can be calculated by fitting a linear curve to the experimental data in Fig. 7b. It is interesting to note that the SRS decreases with increasing annealing temperature. Previous research have indicated that the grain size has strong influence on the SRS of materials, and the SRS of FCC metals and alloys is observed to increase with the reduction of grain size [40]. Based on Figs. 2 and 3 for the microstructures, the average grain/feature size increases with increasing annealing temperature, which can explain the observed decreasing SRS.

The susceptibility for strain localization in metals and alloys was reported to be dependent on the strain hardening exponent $(n)$ and the SRS $(m)$ [58]. For example, the flow instability under uniaxial quasi-static tensile loading can be predicated fairly well by the well-known Considére criterion, the critical necking point for onset of strain localization can be characterized by the following equation:

$\frac{1}{\sigma}\left(\frac{\partial \sigma}{\partial \varepsilon}\right)_{\dot{\varepsilon}} \leq 1$

where $\sigma$ and $\varepsilon$ are the true stress and the true strain, $\dot{\varepsilon}$ is the true strain rate.

While the Hart's criterion can be used to predicate the onset of strain localization when considering strain rate effect (SRS, $m$ ) during uniaxial tensile loading [59]:

$\frac{1}{\sigma}\left(\frac{\partial \sigma}{\partial \varepsilon}\right)_{\dot{\varepsilon}}+m \leq 1$
Staker [60] proposed a formula to estimate the critical shear strain for shear localization under dynamic loading by neglecting the effect of strain-rate hardening:

$\gamma_{C}=\frac{\rho C_{p} n}{\partial \tau / \partial T}$

where $\rho$ is the density, $C_{p}$ is the specific heat capacity, $\frac{\partial \tau}{\partial T}$ is the thermalsoftening parameter. $\rho, C_{p}$, $\frac{\partial \tau}{\partial T}$ can be taken as $8.9 \mathrm{~kg} / \mathrm{m}^{3}, 450 \mathrm{~J} /(\mathrm{kg} \cdot \mathrm{K})$, $-0.4 \mathrm{MPa} / \mathrm{K}$, from the previous paper [42,48], respectively. Eq. (5) characterizes the competition between strain hardening and thermal softening for onset of shear localization.

Learning from the Hart's criterion, we proposed a formula to estimate the critical shear strain for shear localization under dynamic loading when considering the strain-rate hardening effect:

$\gamma_{C}=\frac{\rho C_{p}(n+m)}{\partial \tau / \partial T}$

Therefore, the new formula expresses the competition of strain hardening and strain rate hardening against thermal softening prior to shear strain localization.

The onset of the strain localization under dynamic shear loading was found to be well consistent with the maximum stress point [25], thus the shear strain at the maximum stress point was considered as the critical shear strain for the formation of ASB. Then, the experimental and predicted critical shear strains (Eq. (6)) for onset of shear localization are plotted as a function of annealing temperature in Fig. 7c. It is interesting to note that the predicted results are in a fairly good agreement with the experimental data, indicating that the proposed new formula can predict the critical shear strain very well. Compared to the other metals and alloys [41,42,61-63], it is also shown that this Cantor alloy with CG can postpone shear strain localization to higher levels of shear strain due to
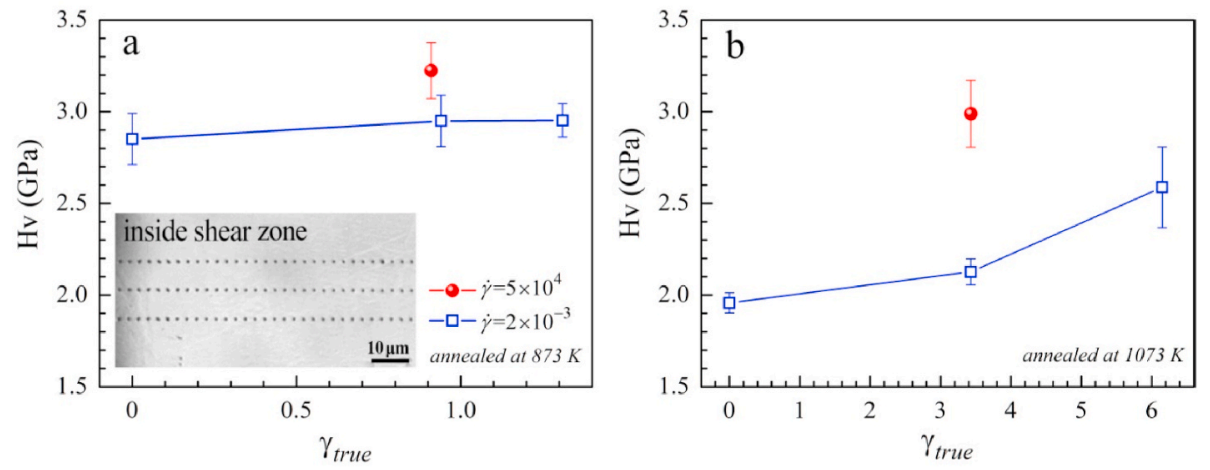

Fig. 8. Average micro-hardness over the shear zone as a function of the interrupted shear strain under quasi-static strain rate $\left(2 \times 10^{-3} \mathrm{~s}^{-1}\right)$ for the samples annealed at (a) $873 \mathrm{~K}$ and (b) $1073 \mathrm{~K}$. The experimental data under high strain rate $\left(5 \times 10^{4} \mathrm{~s}^{-1}\right)$ at the interrupted shear strains of about 0.91 and 3.43 are also included for comparison. 

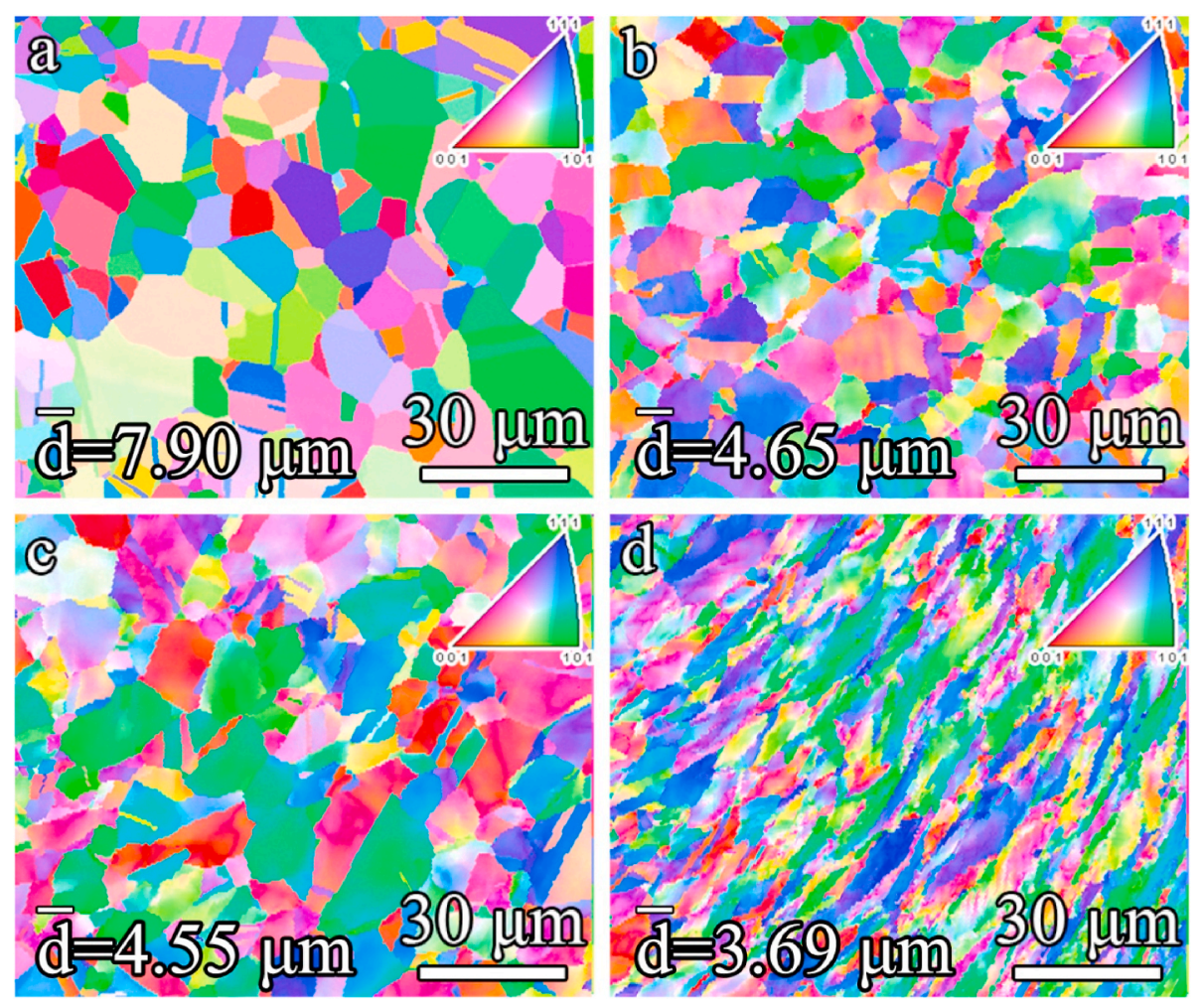

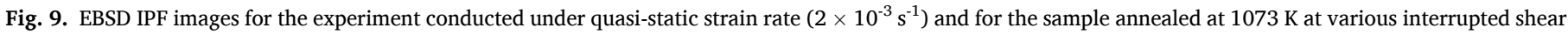
strains: (a) prior to shear loading; (b) at the shear strain of 1.31; (c) at the shear strain of 3.43; (d) at the shear strain of 6.15 .

the exceptional strain hardening ability for CG and the moderate thermal-softening effect.

As indicated in Figs. 5 and 6, apparent strain hardening is observed during shear loading at various strain rates for various microstructures. In this regard, the Vickers micro-hardness $(H v)$ distributions in the shear zone before the shear loading and after shear loading at various interrupted shear strains are measured for the samples annealed at 873 and $1073 \mathrm{~K}$ and the shear tests conducted under both quasi-static strain rate $\left(2 \times 10^{-3} \mathrm{~s}^{-1}\right)$ and high strain rate $\left(5 \times 10^{4} \mathrm{~s}^{-1}\right)$. Thirty points in the shear zone were analyzed, three group of tests were conducted for each point
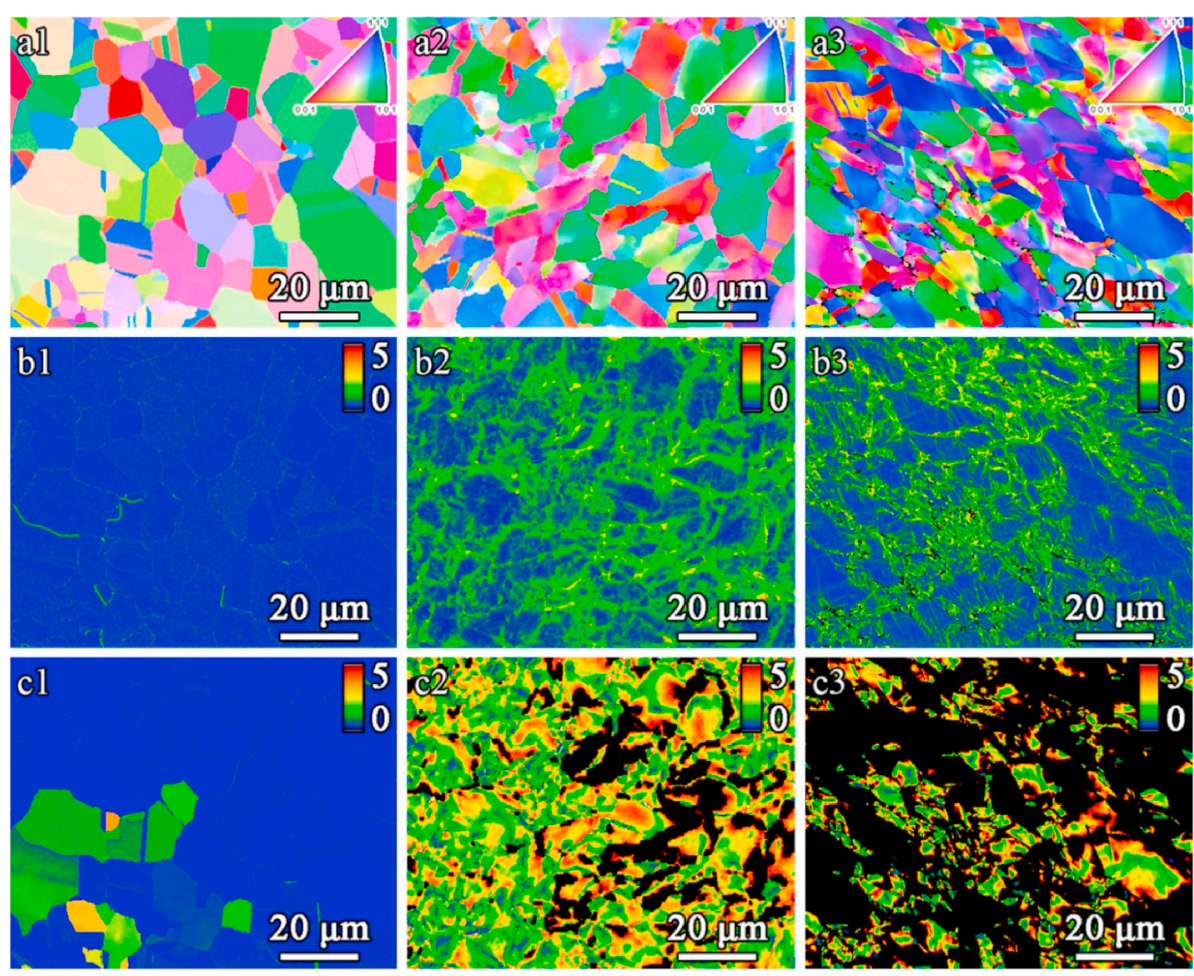

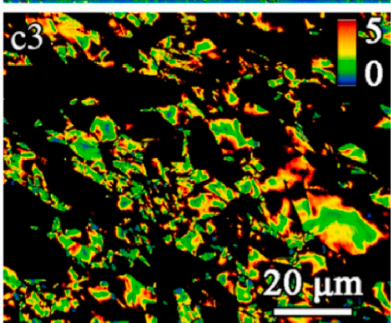

Fig. 10. EBSD IPF images (a1-a3), KAM contours (b1b3) and GROD contours (c1-c3) for the sample annealed at $1073 \mathrm{~K}$ a1-c1 figures are for the microstructure before the shear loading; a2-c2 figures are for the microstructure after quasi-static strain rate (2 $\times 10^{-3} \mathrm{~s}^{-1}$ ) loading at the interrupted shear strain of -3.43; a3-c3 figures are for the microstructure after high strain rate $\left(5 \times 10^{4} \mathrm{~s}^{-1}\right)$ loading at the interrupted shear strain of $\sim 3.43$. In $\mathrm{c} 2$ and $\mathrm{c} 3$, the back color represents the area with the GROD value $>5$. (For interpretation of the references to color in this figure legend, the reader is referred to the Web version of this article.) 
and the error bars were calculated based on these ninety measurements (as shown in the inset of Fig. 8a). Then, the micro-hardness is averaged over the shear zone and the average micro-hardness is plotted as a function of the interrupted shear strain for the samples annealed at 873 and $1073 \mathrm{~K}$ in Fig. $8 \mathrm{a}$ and b, respectively. The micro-hardness increment after shear loading $(\Delta H v)$ can be considered as an indicator of strain hardening ability. It is observed that the average micro-hardness increases with increasing shear strain under quasi-static strain rate for both samples, and the micro-hardness increment is more obvious in the sample annealed at $1073 \mathrm{~K}$ than that in the sample annealed at $873 \mathrm{~K}$, indicating stronger strain hardening for the sample annealed at $1073 \mathrm{~K}$. Moreover, as indicated in Fig. $8 \mathrm{a}$ and b, the micro-hardness increment is more obvious under dynamic shear loading than that under quasi-static loading at the same interrupted shear strains (about 0.91 and 3.43). This indicates that the strain hardening due to the microstructure evolution should be much stronger under dynamic shear loading than that under quasi-static loading, which will be further revealed by EBSD and TEM observations next.

The EBSD IPF images at various interrupted shear strains are displayed in Fig. 9 for the experiment conducted under quasi-static strain rate $\left(2 \times 10^{-3} \mathrm{~s}^{-1}\right)$ and for the sample annealed at $1073 \mathrm{~K}$. As indicated in Fig. 9, the estimated average grain size before the shear loading is about $7.90 \mu \mathrm{m}$, and becomes to about $4.65 \mu \mathrm{m}$ at the shear strain of 1.31 , and becomes to about $4.55 \mu \mathrm{m}$ at the shear strain of 3.43 , and finally is refined to about $3.69 \mu \mathrm{m}$ at the shear strain of 6.15 . The average grain size in Fig. $9 \mathrm{~d}$ was also estimated by intercept method, in which the lines were selected to be perpendicular to the lamellar width. It is also interesting to note that the grains in the shear zone are sheared and elongated along the shear direction at the shear strain of 6.15 . The grains are significantly refined during the uniform shear deformation, thus the strong strain hardening under shear deformation should be mainly attributed to the so-called "dynamic Hall-Petch effect" induced by the grain refinement [25].

In order to compare the microstructures before the shear loading, for the experiments conducted at both quasi-static strain rate $\left(2 \times 10^{-3} \mathrm{~s}^{-1}\right)$ and high strain rate $\left(5 \times 10^{4} \mathrm{~s}^{-1}\right)$ at the same interrupted shear strain $(\sim 3.43)$, the IPF images (Fig. 10a1-10a3), the Kernel average misorientation (KAM) contours (Fig. 10b1-10b3) and the grain reference orientation deviation (GROD) contours (Fig. 10c1-10c3) for the sample annealed at $1073 \mathrm{~K}$ are shown in Fig. 10. In general, KAM values represent the geometrically necessary dislocation (GND) density in the sample [64], while GROD values are a kind of grain lattice distortion parameter which can be linearly related to the nominal plastic strain of representative volume element RVE containing enough grains under mechanical loading [65]. It is observed that the grain refinement is more severe under dynamic loading (down to $3.89 \mu \mathrm{m}$ ) than that under quasi-static loading (down to $4.55 \mu \mathrm{m}$ ) at the same interrupted shear strain. This means that the stronger strain hardening by microstructure
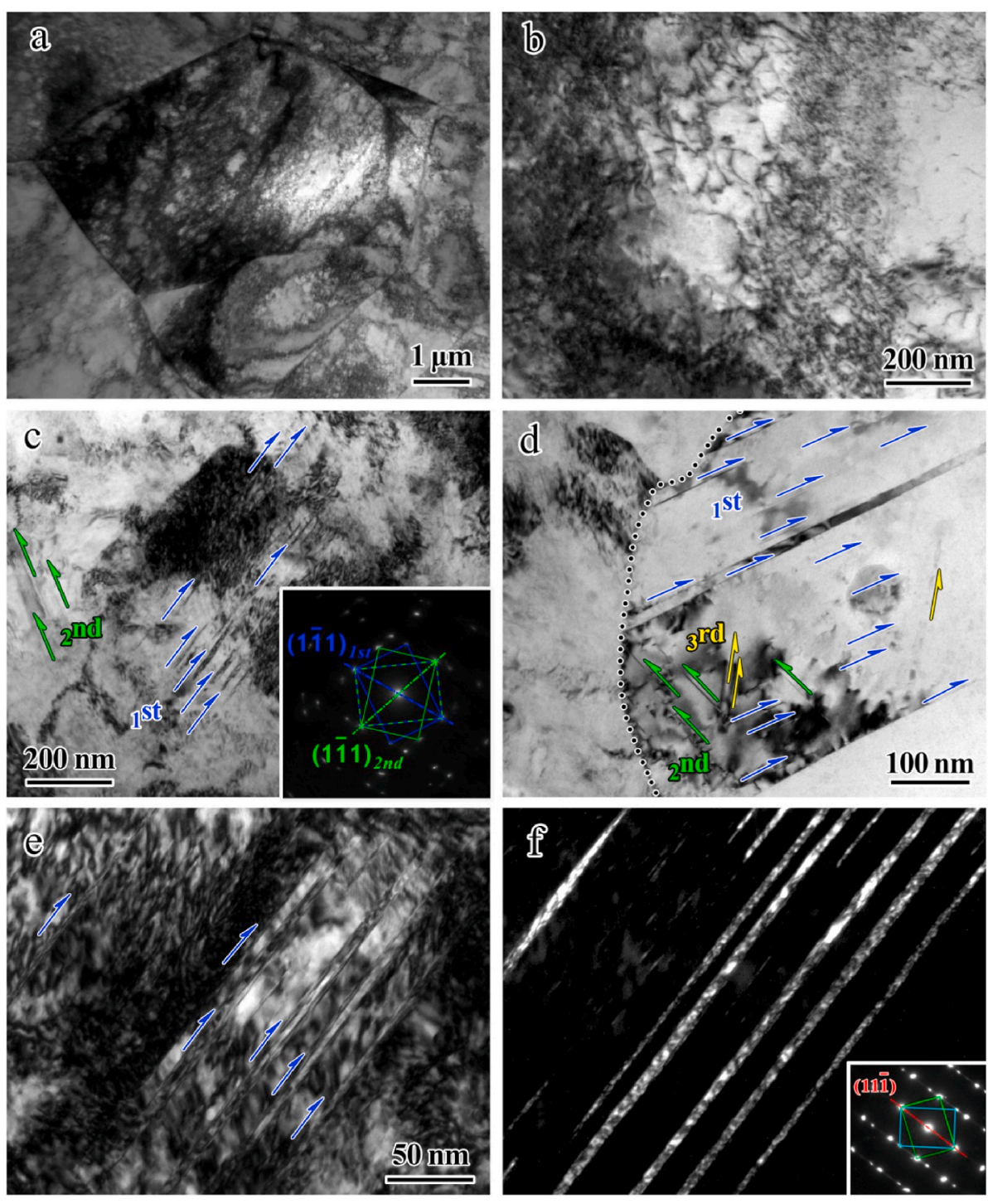

Fig. 11. TEM images for the experiments conducted: (a) and (b) at quasi-static strain rate $\left(2 \times 10^{-3} \mathrm{~s}^{-1}\right)$; (c)-(f) high strain rate $\left(5 \times 10^{4} \mathrm{~s}^{-1}\right)$. These images are taken for the sample annealed at $1073 \mathrm{~K}$ at the same interrupted shear strain $(-3.43)$. Hierarchical nanotwins $\left(1^{\text {st }}\right.$ for primary twins, $2^{\text {nd }}$ for secondary twins, $3^{\text {rd }}$ for tertiary twins) are indicated in (c) and (d). Images of (a)-(e) are bright-field images, while image (f) is dark-field image for image (e). The selected area diffraction for inset of (f) clearly indicates the twin relationship. 
evolution under dynamic loading can be attributed to the more obvious "dynamic Hall-Petch effect", which is consistent with the aforementioned micro-hardness results. Before shear loading, both KAM and GROD values are very low due to high temperature annealing. However, KAM values become much higher after quasi-static and dynamic deformations, indicating high density of GNDs for accommodating deformation incompatibility. It is also interesting to note that the area with high GROD value $(>5)$ is much larger after dynamic loading than that after quasi-static loading at the same interrupted shear strain, indicating that the plastic strain is more concentrated and the strain partitioning is more obvious under dynamic loading.

TEM bright-field images for the sample annealed at $1073 \mathrm{~K}$ and for the experiments conducted at both quasi-static strain rate $\left(2 \times 10^{-3} \mathrm{~s}^{-1}\right)$ and high strain rate $\left(5 \times 10^{4} \mathrm{~s}^{-1}\right)$ at the same interrupted shear strain $(\sim 3.43)$ are displayed in Fig. 11. High density of dislocations are observed after quasi-static deformation, and dislocation tangles, dislocation cells and dislocation walls are also observed in the grain interiors. While, no deformation twins (DTs) are formed under quasi-static deformation. In contrast, deformation twins and multiple deformation twins have been observed in the grain interiors under dynamic shear loading (Fig. 11c-f). It was reported that multiple deformation twins with different orientations can further improve the mechanical properties in metals and alloys when compared to the single deformation twins, since hierarchical nanotwins are more effective in obstructing dislocation motion and in accumulating dislocations [66]. The multiple twinning network in hierarchical nanotwins can provide more barriers for dislocation slip to result in higher strength, and the multiple network in different orientations can also present adequate pathways for easy glide and cross-slip of dislocations to accommodate significant plastic deformation for stronger strain hardening $[66,67]$. Thus, the higher micro-hardness increment and the higher strain hardening due to the microstructure evolution under dynamic shear loading can be partly attributed to the transition of deformation mechanism [68], i.e., from the dislocation slip under quasi-static strain rate to the triggered deformation twins and the multiple hierarchical nanotwins under high strain rate. It also should be noted that the nanotwinned alloys generally display remarkable SRS, which also helps for strain hardening and ductility under high strain rate [69].

\section{Summary and concluding remarks}

Strain rate effects on the shear deformation behaviors of the CrMnFeCoNi HEA with various heterogeneous grain structures have been revealed by shear testing at a wide range of strain rates $\left(2 \times 10^{-3}-5\right.$ $\times 10^{4} \mathrm{~s}^{-1}$ ) and subsequent microstructure observations, the main findings and conclusions can be summarized as follows:

(1) Under lower strain rates $\left(2 \times 10^{-3}, 1 \times 10^{-1}, 1 \times 10^{1} \mathrm{~s}^{-1}\right)$, the microstructures annealed at $873,948,973,1073 \mathrm{~K}$ display strong strain hardening, while the CR sample and the microstructure annealed at 848 $\mathrm{K}$ show slight strain hardening. It is obviously observed that the shear yield strength decreases and the strain hardening ability increases with increasing annealing temperature. Moreover, the shear stress-strain curves under the dynamic shear testing $\left(5 \times 10^{4} \mathrm{~s}^{-1}\right)$ show much lower strain hardening ability for each microstructure when compared to those conducted under the other lower strain rates, possibly due to the adiabatic heating and the thermal softening effect. Based on uniaxial tensile testing and shear testing results, strain hardening exponent $(n)$ and strain rate sensitivity $(m)$ have been obtained for various microstructures.

(2) Under lower strain rates $\left(2 \times 10^{-3}-1 \times 10^{1} \mathrm{~s}^{-1}\right)$, shear localization was not observed up to shear strain of 8 for all microstructures. However, stress drop and shear localization were found to occur at various critical shear strains for various microstructures under dynamic shear loading $\left(5 \times 10^{4} \mathrm{~s}^{-1}\right)$. The critical shear strain was found to increase with increasing annealing temperature. Based on the competition of strain hardening and strain rate hardening against thermal softening prior to shear localization, a new formula was proposed to estimate the critical shear strains under dynamic shear loading and the predicted results were observed to be in a fairly good agreement with the experimental data.

(3) The micro-hardness increment is observed to be more obvious under dynamic shear loading than that under quasi-static strain rate at the same interrupted shear strain $(\sim 3.43)$. This indicates that the strain hardening due to the microstructure evolution should be much stronger under dynamic shear loading than that under quasi-static loading, which can be attributed to the more efficient grain refinement and the triggered hierarchical deformation twins under dynamic shear deformation. The present findings should provide insights for understanding the deformation mechanisms of HEAs with heterogeneous grain structures under a wide range of strain rates.

\section{Data availability}

The raw/processed data required to reproduce these findings cannot be shared at this time due to technical or time limitations.

\section{Declaration of competing interest}

The authors declare that they have no known competing financial interests or personal relationships that could have appeared to influence the work reported in this paper.

\section{CRediT authorship contribution statement}

Zhengling Yang: Investigation. Muxin Yang: Investigation. Yan Ma: Investigation. Lingling Zhou: Investigation. Wenqiang Cheng: Investigation. Fuping Yuan: Conceptualization, Writing - original draft, Supervision. Xiaolei Wu: Conceptualization, Supervision.

\section{Acknowledgements}

The work was supported by the National Key R\&D Program of China [grant number 2017YFA0204402]; the National Natural Science Foundation of China [grant numbers 11672313 and 11790293], and the Strategic Priority Research Program of the Chinese Academy of Sciences [grant number XDB22040503].

\section{References}

[1] J.W. Yeh, S.K. Chen, S.J. Lin, J.Y. Gan, T.S. Chin, T.T. Shun, C.H. Tsau, S.Y. Chang, Nanostructured high-entropy alloys with multiple principal elements: novel alloy design concepts and outcomes, Adv. Eng. Mater. 6 (2004) 299-303.

[2] B. Cantor, I.T.H. Chang, P. Knight, A.J.B. Vincent, Microstructural development in equiatomic multicomponent alloys, Mater. Sci. Eng., A 375 (2004) 213-218.

[3] F. Otto, A. Dlouhý, C. Somsen, H. Bei, G. Eggeler, E.P. George, The influences of temperature and microstructure on the tensile properties of a CoCrFeMnNi highentropy alloy, Acta Mater. 61 (2013) 5743-5755.

[4] B. Gludovatz, A. Hohenwarter, D. Catoor, E.H. Chang, E.P. George, R.O. Ritchie, A fracture-resistant high-entropy alloy for cryogenic applications, Science 345 (2014) 1153-1158.

[5] Y. Zhang, T.T. Zuo, Z. Tang, M.C. Gao, K.A. Dahmen, P.K. Liaw, Z.P. Lu, Microstructures and properties of high-entropy alloys, Prog. Mater. Sci. 61 (2014) $1-93$.

[6] B. Schuh, F. Mendez-Martin, B. Völker, E.P. George, H. Clemens, R. Pippan, A. Hohenwarter, Mechanical properties, microstructure and thermal stability of a nanocrystalline CoCrFeMnNi high-entropy alloy after severe plastic deformation, Acta Mater. 96 (2015) 258-268.

[7] Y.F. Ye, Q. Wang, J. Lu, C.T. Liu, Y. Yang, High-entropy alloy: challenges and prospects, Mater. Today 19 (2015) 349-362.

[8] Y. Zhang, T.T. Zuo, Z. Tang, M.C. Gao, K.A. Dahmen, P.K. Liaw, Z.P. Lu, Microstructures and properties of high-entropy alloys, Prog. Mater. Sci. 20 (2017) 293-300.

[9] M.H. Tsai, J.W. Yeh, High-entropy alloys: a critical review, Mater. Res. Lett. 2 (2014) 107-123.

[10] D.B. Miracle, O.N. Senkov, A critical review of high entropy alloys and related concepts, Acta Mater. 122 (2017) 448-511.

[11] E.P. George, D. Raabe, R.P. Ritchie, High-entropy alloys, Nat. Rev. Mater. 4 (2019) $515-534$. 
[12] Z. Li, K.G. Pradeep, Y. Deng, D. Raabe, C.C. Tasan, Metastable high-entropy dualphase alloys overcome the strength-ductility trade-off, Nature 534 (2016) $227-230$.

[13] Y.H. Jo, S. Jung, W.M. Choi, S.S. Sohn, H.S. Kim, B.J. Lee, N.J. Kim, S. Lee, Cryogenic strength improvement by utilizing room-temperature deformation twinning in a partially recrystallized VCrMnFeCoNi high-entropy alloy, Nat. Commun. 8 (2017) 15719.

[14] Z.F. Lei, X.J. Liu, Y. Wu, H. Wang, S.H. Jiang, S.D. Wang, X.D. Hui, Y.D. Wu, B. Gault, P. Kontis, Enhanced strength and ductility in a high-entropy alloy via ordered oxygen complexes, Nature 563 (2018) 546-550.

[15] T. Yang, Y.L. Zhao, Y. Tong, Z.B. Jiao, J. Wei, J.X. Cai, X.D. Han, D. Chen, A. Hu, J. J. Kai, Multicomponent intermetallic nanoparticles and superb mechanical behaviors of complex alloys, Science 362 (2018) 933-937.

[16] Y.J. Liang, L.J. Wang, Y.R. Wen, B.Y. Cheng, Q.L. Wu, T.Q. Cao, Q. Xiao, Y.F. Xue, G. Sha, Y.D. Wang, High-content ductile coherent nanoprecipitates achieve ultrastrong high-entropy alloys, Nat. Commun. 9 (2018) 4063.

[17] L.J. Santodonato, P.K. Liaw, R.R. Unocic, H. Bei, J.R. Morris, Predictive multiphase evolution in Al-containing high-entropy alloys, Nat. Commun. 9 (2018) 4520.

[18] P.J. Shi, W.L. Ren, T.X. Zheng, Z.M. Ren, X.L. Hou, J.C. Peng, P.F. Hu, Y.F. Gao, Y. B. Zhong, P.K. Liaw, Enhanced strength-ductility synergy in ultrafine-grained eutectic high-entropy alloys by inheriting microstructural lamellae, Nat. Commun. 10 (2019) 489.

[19] Q.Q. Ding, Y. Zhang, X. Chen, X.Q. Fu, D.K. Chen, S.J. Chen, L. Gu, F. Wei, H.B. Bei, Y.F. Gao, M.R. Wen, J.X. Li, Z. Zhang, T. Zhu, R.O. Ritchie, Q. Yu, Tuning element distribution, structure and properties by composition in high-entropy alloys, Nature 574 (2019) 223-227.

[20] B. Gludovatz, A. Hohenwarter, K.V. Thurston, H. Bei, Z. Wu, E.P. George, R. O. Ritchie, Exceptional damage-tolerance of a medium-entropy alloy CrCoNi at cryogenic temperatures, Nat. Commun. 7 (2016) 10602.

[21] Z.J. Zhang, H.W. Sheng, Z.J. Wang, B. Gludovatz, Z. Zhang, E.P. George, Q. Yu, S. X. Mao, R.O. Ritchie, Dislocation mechanisms and 3D twin architectures generate exceptional strength-ductility-toughness combination in CrCoNi medium-entropy alloy, Nat. Commun. 8 (2017) 14390.

[22] S. Yoshida, T. Bhattacharjee, Y. Bai, N. Tsuji, Friction stress and Hall-Petch relationship in CoCrNi equi-atomic medium entropy alloy processed by severe plastic deformation and subsequent annealing, Scripta Mater. 134 (2017) 33-36.

[23] J. Miao, C.E. Slone, T.M. Smith, C. Niu, H. Bei, M. Ghazisaeidi, G.M. Pharr, M. J. Mills, The evolution of the deformation substructure in a Ni-Co-Cr equiatomic solid solution alloy, Acta Mater. 132 (2017) 35-48.

[24] M.X. Yang, D.S. Yan, F.P. Yuan, P. Jiang, E. Ma, X.L. Wu, Dynamically reinforced heterogeneous grain structure prolongs ductility in a medium-entropy alloy with gigapascal yield strength, Proc. Natl. Acad. Sci. U.S.A. 115 (2018) 7224-7229.

[25] Y. Ma, F.P. Yuan, M.X. Yang, P. Jiang, E. Ma, X.L. Wu, Dynamic shear deformation of a CrCoNi medium-entropy alloy with heterogeneous grain structures, Acta Mater. 148 (2018) 407-418.

[26] J. Ding, Q. Yu, M. Asta, R.O. Ritchie, Tunable stacking fault energies by tailoring local chemical order in CrCoNi medium-entropy alloys, Proc. Natl. Acad. Sci. U.S. A. 115 (2018) 8919-8924.

[27] C.E. Slone, J. Miao, E.P. George, M.J. Mills, Achieving ultra-high strength and ductility in equiatomic CrCoNi with partially recrystallized microstructures, Acta Mater. 165 (2019) 496-507.

[28] M.X. Yang, L.L. Zhou, C. Wang, P. Jiang, F.P. Yuan, E. Ma, X.L. Wu, High impact toughness of CrCoNi medium-entropy alloy at liquid-helium temperature, Scripta Mater. 172 (2019) 66-71.

[29] Q.-J. Li, H. Sheng, E. Ma, Strengthening in multi-principle element alloys with local-chemical-order roughened dislocation pathways, Nat. Commun. 10 (2019) 3563.

[30] F.P. Yuan, W.Q. Cheng, S.D. Zhang, X.M. Liu, X.L. Wu, Atomistic simulations of tensile deformation in a CrCoNi medium-entropy alloy with heterogeneous grain structures, Materialia 9 (2020) 100565.

[31] X.L. Wu, M.X. Yang, P. Jiang, C. Wang, L.L. Zhou, F.P. Yuan, E. Ma, Deformation nanotwins suppress shear banding during impact test of CrCoNi medium-entropy alloy, Scripta Mater. 178 (2020) 452-456.

[32] Z. Wu, H. Bei, F. Otto, G.M. Pharr, E.P. George, Recovery, recrystallization, grain growth and phase stability of a family of FCC-structured multi-component equiatomic solid solution alloys, Intermetallics 46 (2014) 131-140.

[33] Y.L. Zhao, T. Yang, Y. Tong, J. Wang, J.H. Luan, Z.B. Jiao, D. Chen, Y. Yang, A. Hu, C.T. Liu, J.J. Kai, Heterogeneous precipitation behavior and stackingfaultmediated deformation in a CoCrNi-based medium-entropy alloy, Acta Mater. 138 (2017) 72-82.

[34] S.S. Sohn, A.K. da Silva, Y. Ikeda, F. Kormann, W.J. Lu, W.S. Choi, B. Gault, D. Ponge, J. Neugebauer, Ultrastrong medium-rntropy single-phase alloys designed via severe lattice distortion, Adv. Mater. 31 (2019) 1807142.

[35] X.L. Wu, P. Jiang, L. Chen, F.P. Yuan, Y.T. Zhu, Extraordinary strain hardening by gradient structure, Proc. Natl. Acad. Sci. U.S.A. 111 (2014) 7197-7201.

[36] X.L. Wu, M.X. Yang, F.P. Yuan, G.L. Wu, Y.J. Wei, X.X. Huang, Y.T. Zhu, Heterogeneous lamella structure unites ultrafine-grain strength with coarse-grain ductility, Proc. Natl. Acad. Sci. U.S.A. 112 (2015) 14501-14505.

[37] Y.T. Zhu, X.L. Wu, Perspective on hetero-deformation induced (HDI) hardening and back stress, Mater. Res. Lett. 7 (2019) 393-398.

[38] M.A. Meyers, Dynamic Behavior of Materials, Wiley-Interscience, New York, USA, 1994, pp. 323-326.
[39] Y.Z. Guo, Q.C. Ruan, S.X. Zhu, Q. Wei, H.S. Chen, J.N. Lu, B. Hu, X.H. Wu, Y.L. Li, D.N. Fang, Temperature rise associated with adiabatic shear band: causality clarified, Phys. Rev. Lett. 122 (2019), 015503.

[40] Q. Wei, Strain rate effects in the ultrafine grain and nanocrystalline regimes influence on some constitutive responses, J. Mater. Sci. 42 (2007) 1709-1727.

[41] A. Mishra, M. Martin, N.N. Thadhani, B.K. Kad, E.A. Kenik, M.A. Meyers, Highstrain rate response of ultra-fine-grained copper, Acta Mater. 56 (2008) 2770-2783.

[42] Z.Z. Li, S.T. Zhao, S.M. Alotaibi, Y. Liu, B.F. Wang, M.A. Meyers, Adiabatic shear localization in the CrMnFeCoNi high-entropy alloy, Acta Mater. 151 (2018) 424-431.

[43] J.Y. He, Q. Wang, H.S. Zhang, L.H. Dai, T. Mukai, Y. Wu, X.J. Liu, H. Wang, T.G. Nieh, Z.P. Lu, Dynamic deformation behavior of a face-centered cubic FeCoNiCrMn high-entropy alloy, Sci. Bull. 63 (2018) 362-368.

[44] T.W. Zhang, Z.M. Jiao, Z.H. Wang, J.W. Qiao, Dynamic deformation behaviors and constitutive relations of an AlCoCr1.5Fe1.5NiTi0.5 high-entropy alloy, Scripta Mater. 136 (2017) 15-19.

[45] L. Tian, Z.M. Jiao, G.Z. Yuan, S.G. Ma, Z.H. Wang, H.J. Yang, Y. Zhang, J.W. Qiao, Effect of strain rate on deformation behavior of AlCoCrFeNi high-entropy alloy by nanoindentation, J. Mater. Eng. Perform. 25 (2016) 2255-2260.

[46] Y.H. Jo, D.G. Kim, M.C. Jo, K.-Y. Doh, S.S. Sohn, D. Lee, H.S. Kim, B.J. Lee, S. Lee, Effects of deformation-induced BCC martensitic transformation and twinning on impact toughness and dynamic tensile response in metastable VCrFeCoNi highentropy alloy, J. Alloys Compd. 785 (2019) 1056-1067.

[47] S. Gangireddy, B. Gwalani, R.S. Mishra, Grain size dependence of strain rate sensitivity in a single phase FCC high entropy alloy Al0.3CoCrFeNi, Mater. Sci. Eng., A 736 (2018) 344-348.

[48] Z. Li, S. Zhao, H. Diao, P.K. Liaw, M.A. Meyers, High-velocity deformation of Al0.3CoCrFeNi high-entropy alloy: remarkable resistance to shear failure, Sci. Rep. 7 (2017) 42742.

[49] J. Qiu, G.S. Xiao, T. Jin, B.Y. Su, X.F. Shu, S.G. Ma, Indentation strain rate sensitivity of CoCrFeNiAl0.3 high-entropy alloy, Adv. Eng. Mater. 21 (2019) 1800744.

[50] L. Wang, J.W. Qiao, S.G. Ma, Z.M. Jiao, T.W. Zhang, G. Chen, D. Zhao, Y. Zhang, Z. $\mathrm{H}$. Wang, Mechanical response and deformation behavior of Al0.6CoCrFeNi highentropy alloys upon dynamic loading, Mater. Sci. Eng., A 727 (2018) 208-213.

[51] S. Gangireddy, L. Kaimiao, B. Gwalani, R. Mishra, Microstructrual dependence of strain rate sensitivity in thermomechanically processed Al0.1 CoCrFeNi high entropy alloy, Mater. Sci. Eng., A 727 (2018) 148-159.

[52] S. Gangireddy, B. Gwalani, K.M. Liu, R. Banerjee, R.S. Mishra, Microstructures with extraordinary dynamic work hardening and strain rate sensitivity in Al0.3CoCrFeNi high entropy alloy, Mater. Sci. Eng., A 734 (2018) 42-50.

[53] M. Komarasamy, K. Alagarsamy, R.S. Mishra, Serration behavior and negative strain rate sensitivity of Al0.1CoCrFeNi high entropy alloy, Intermetallics 84 (2017) 20-24.

[54] J.M. Park, J. Moon, J.W. Bae, M.J. Jang, J. Park, S. Lee, H.S. Kim, Strain rate effects of dynamic compressive deformation on mechanical properties and microstructure of CoCrFeMnNi high-entropy alloy, Mater. Sci. Eng., A 719 (2018) 155-163.

[55] S.G. Ma, Z.M. Jiao, J.W. Qiao, H.J. Yang, Y. Zhang, Z.H. Zhang, Strain rate effects on the dynamic mechanical properties of the AlCrCuFeNi2 high-entropy alloy, Mater. Sci. Eng., A 649 (2016) 35-38.

[56] D.Y. Li, Y. Zhang, The ultrahigh charpy impact toughness of forged AlxCoCrFeNi high entropy alloys at room and cryogenic temperatures, Intermetallics 70 (2016) 24-28.

[57] J.H. Sung, J.H. Kim, R.H. Wagoner, A plastic constitutive equation incorporating strain, strain-rate, and temperature, Int. J. Plast. 26 (2010) 1746-1771.

[58] T.W. Wright, Shear band susceptibility - work-hardening materials, Int. J. Plast. 8 (1992) 583-602.

[59] E.W. Hart, Theory of the tensile test, Acta Metall. 15 (1967) 351-355.

[60] M.R. Staker, The relation between adiabatic shear instability strain and material properties, Acta Metall. 29 (1981) 683-689.

[61] M.A. Meyers, G. Subhash, B.K. Kad, L. Prasad, Evolution of microstructure and shear-band formation in a-hcp titanium, Mech. Mater. 17 (1994) 175-193.

[62] Q. Xue, M.A. Meyers, V.F. Nesterenko, Self-organization of shear bands in titanium and Ti-6Al-4V alloy, Acta Mater. 50 (2002) 575-596.

[63] G.R. Johnson, J.M. Hoegfeldt, U.S. Lindholm, A. Nagy, Response of various metals to large torsional strains over a large range of strain rates - Part 1: ductile metals, J. Eng. Mater. Technol. 105 (1983) 42-47.

[64] L.P. Kubin, A. Mortensen, Geometrically necessary dislocations and strain gradient plasticity: a few critical issues, Scripta Mater. 48 (2003) 119-125.

[65] S.-S. Rui, Y.-B. Shang, Y.-N. Fan, Q.-N. Han, L.-S. Niu, H.-J. Shi, K. Hashimoto, N. Komai, EBSD analysis of creep deformation induced grain lattice distortion: a new method for creep damage evaluation of austenitic stainless steels, Mater. Sci. Eng., A 733 (2018) 329-337.

[66] X.W. Liu, L.G. Sun, L.L. Zhu, J.B. Liu, K. Lu, J. Lu, High-order hierarchical nanotwins with superior strength and ductility, Acta Mater. 149 (2018) 397-406.

[67] H.N. Kou, J. Lu, Y. Li, High-strength and high-ductility nanostructured and amorphous metallic materials, Adv. Mater. 26 (2014) 5518-5524.

[68] W.Y. Huo, F. Fang, X.D. Liu, S.Y. Tan, Z.H. Xie, J.Q. Jiang, Remarkable strain-rate sensitivity of nanotwinned CoCrFeNi alloys, Appl. Phys. Lett. 114 (2019) 101904.

[69] W.Y. Huo, H. Zhou, F. Fang, X.J. Hu, Z.H. Xie, J.Q. Jiang, Strain-rate effect upon the tensile behavior of CoCrFeNi high-entropy alloys, Mater. Sci. Eng., A 689 (2017) 366-369. 